\title{
THE FREE TOPOLOGICAL GROUP ON A SIMPLY CONNECTED SPACE
}

\author{
J. P. L. HARDY AND SIDNEY A. MORRIS
}

\begin{abstract}
It is shown that the free $k$-group on a simply connected locally equiconnected space is simply connected. This result is then used to verify, for a large class of groups, the conjecture of Ordman that $\pi_{1}(G * H)$ $=\pi_{1}(G) \times \pi_{1}(H)$, where $G * H$ is the free product of $G$ and $H$.
\end{abstract}

1. Preliminaries. We begin by recalling a few definitions. A topological space $X$ is said to be a $k$-space if a subset $U$ of $X$ is closed in $X$ whenever $f^{-1}(U)$ is closed in $C$, for each compact Hausdorff space $C$ and each continuous map $f: C \rightarrow X$. Further, $X$ is said to be $k$-Hausdorff if $f(C)$ is closed in $X$, for each $C$ and each $f$. Throughout the paper we will work in the category $\mathscr{K}$ of $k$-Hausdorff $k$-spaces and continuous maps. In particular, $X$ will always denote a $k$-Hausdorff $k$-space.

We define a $k$-group $G$ to be a group whose underlying space is a $k$-Hausdorff $k$-space and whose composition $\theta: G \times{ }_{k} G \rightarrow G$ and inverse $\sigma$ : $G \rightarrow G$ are continuous. ( $G \times{ }_{k} G$ denotes the product in $\mathscr{K}$ of two copies of $G$.) The free $k$-group [11], [7], [6] on a pointed space $(X, e)$ consists of a $k$-group $F(X)$ and a continuous pointed map $i: X \rightarrow F(X)$ with the property that for any $k$-group $G$ and any continuous pointed map $f$ of $X$ into $G$, there exists a unique continuous homomorphism $f^{*}$ of $F(X)$ into $G$ such that $f^{*} i=f$. It is proved in Ordman [11] and Hardy [7] that $F(X)$ always exists, is independent of the choice of base point, and is algebraically the free group on $i(X \backslash\{e\})$. Further, $i$ maps $X$ homeomorphically onto a closed subspace of $F(X)$; so we regard $X$ itself as a closed subspace of $F(X)$.

Hardy [7, Chapter V, Theorem 3.1] shows that $F(X)$ is an iterated adjunction space [1]. (This can also be deduced from [11, §2] using [3, 4.5.8].) More precisely, if $F_{n}(X)$ denotes the closed subset of $F(X)$ comprising all the words of length at most $n$, then $F(X)$ has the weak topology with respect to the $F_{n}(X)$ and each $F_{n}(X)$ is an adjunction space as follows. Let $X^{-1}$ be a homeomorphic copy of $X$ with elements $x^{-1}$ for each $x \in X$, and let $\bar{X}$ be the one-point union $X \vee X^{-1}$. Further, let $(\bar{X})^{n}$ denote the product in $\mathscr{K}$ of $n$ copies of $\bar{X}$ and $p_{n}:(\bar{X})^{n} \rightarrow F_{n}(X)$ the map $\left(x_{1}^{\varepsilon_{1}}, \ldots, x_{n}^{\varepsilon_{n}}\right) \rightarrow x_{1}^{\varepsilon_{1}} \ldots x_{n}^{\varepsilon_{n}}$, where each $x_{i} \in X$ and $\varepsilon_{i}= \pm 1$. Then $F_{n}(X)$ is the adjunction spaces $F_{n-1}(X) \cup \cup_{f_{n-1}}(\bar{X})^{n}$, where the attaching $\operatorname{map} f_{n-1}$ is the restriction of $p_{n}$ to the closed subset $A_{n-1}(X)=p_{n}^{-1}\left(F_{n-1}(X)\right)$ of $(\bar{X})^{n}$.

Received by the editors September 10, 1974.

AMS (MOS) subject classifications (1970). Primary 57F20, 55A05; Secondary 55D05, 20E05, $54 \mathrm{C} 55$

Key words and phrases. Free topological group, $k$-group, simply connected, free product of $k$-groups, locally equiconnected, cofibration, van Kampen theorem, fundamental groupoid.

(c) American Mathematical Society 1976 
For our theorem we will need to assume a mild homotopy extension condition. A space $X$ is said to be locally equiconnected (LEC)[5], [4] if the inclusion of the diagonal $\Delta \subset X \times X$ is a closed cofibration. As examples we mention that every metric absolute neighbourhood retract (ANR) and every cell complex is LEC [5]. It is shown in [7] that if $X$ is LEC then the inclusion $A_{n-1}(X) \subset(\bar{X})^{n}$ is a closed cofibration.

Our final recollection is that $X$ is said to be simply connected [3] if each component of its fundamental groupoid is a tree groupoid [8]. Notice that a simply connected space need not be path connected.

\section{Results.}

THEOREM. Let $X$ be an LEC space. If $X$ is simply connected then $F(X)$ is simply connected.

Proof. We shall prove by induction, that each $F_{n}(X)$ is simply connected. Since $F(X)$ has the weak topology with respect to the $F_{n}(X)$, the required result is then a trivial consequence of Lemma 9.3 of [13].

Clearly $F_{1}(X)=X \vee X^{-1}$ is simply connected. Assume $F_{n-1}(X)$ is simply connected. Consider the diagram where $i$ and $j$ are inclusions.

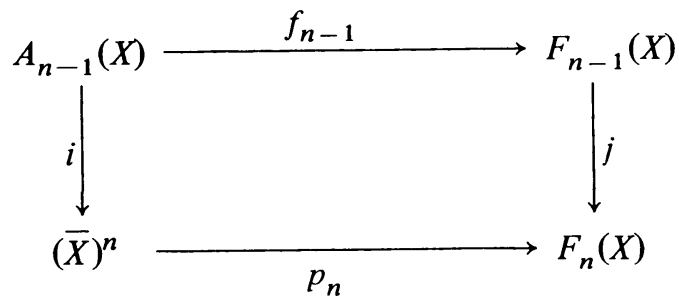

By our preliminary remarks this diagram is a pushout and the inclusion $A_{n-1}(X) \subset F_{n}(X)$ is a closed cofibration. So we can now employ the full strength of the Brown-van Kampen theorem [2], [3]. In so doing we must find "representative" subsets $B, C$ and $D$ of $F_{n}(X),(\bar{X})^{n}$ and $F_{n-1}(X)$, respectively; the set $C$ is also required to be representative in $A_{n-1}(X)$. (A set $A$ is said to be representative in $Z$ if $A \cap Z$ meets each path component of $Z$.)

Let $D$ consist of one point from each path component of $F_{n-1}(X)$. Since $f_{n-1}$ is onto we can choose $C^{\prime}$ to be a subset of $f_{n-1}^{-1}(D)$ comprising one point from each path component of $A_{n-1}(X)$. Finally let $C$ be $C^{\prime}$ together with one point from each path component of $(\bar{X})^{n}$ not represented by $C^{\prime}$.

In accordance with [3] we put $B=p_{n}(C)$ and so obtain that $B, C$ and $D$ are representative, as required. Thus the above square induces the following pushout diagram in the category of groupoids

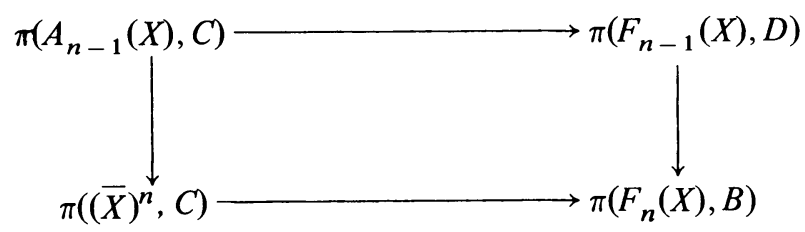

(where $\pi(Z, A)$ denotes the restriction of the fundamental groupoid of $Z$ to 
$A)$. To complete the proof we only have to show that $\pi\left(F_{n-1}(X), D\right)$, $\pi\left(A_{n-1}(X), C\right)$ and $\pi\left((\bar{X})^{n}, C\right)$ are discrete groupoids [8]; that is their only arrows are identity arrows. But we already have that the first two of these are discrete.

Of course $\pi\left((\bar{X})^{n}, C\right)$ is discrete if and only if $C$ meets each path component of $(\bar{X})^{n}$ in precisely one point. Clearly this is the case if distinct path components of $A_{n-1}(X)$ lie in distinct path components of $(\bar{X})^{n}$. Indeed, while it is not in general true that the path components of a subspace are formed by intersecting that subspace with the path components of the whole space, this is so for the subspace $A_{n-1}(X)$ of $(\bar{X})^{n}$. Verifying this is rather tedious (but routine) so we include here only a typical case.

Consider $a \in A_{n-1}(X)$, where

$$
a=\left(x_{1}^{\varepsilon_{1}}, \ldots, x_{i-3}^{\varepsilon_{i-3}}, x_{i-2}, x_{i-2}^{-1}, x_{i}, e, e, x_{i+3}^{\varepsilon_{i+3}}, e, x_{i+5}^{\varepsilon_{i+5}}, \ldots, x_{n}^{\varepsilon_{n}}\right),
$$

with no $x_{j}$ in the path component in $\bar{X}$ of $e$, and no $x_{j+1}^{\xi_{j}+1}$ in the path component in $\bar{X}$ of $x_{j}^{-\varepsilon_{j}}$, but $x_{i}$ and $x_{i-2}$ in the same path component in $\bar{X}$. Let $C_{j}^{\varepsilon_{j}}$ denote the path component in $\bar{X}$ of $x_{j}^{\varepsilon_{j}}, C_{0}$ the path component in $\bar{X}$ of $e$ and $\Delta_{j}^{\varepsilon_{j}}$ the set $C_{j}^{\varepsilon_{j}} \times C_{j}^{-\varepsilon_{j}} \cap\left\{\left(x, x^{-1}\right): x \in \bar{X}\right\}$. Then the path component in $(\bar{X})^{n}$ of $a$ is

$$
\begin{aligned}
C_{a}= & C_{1}^{\varepsilon_{1}} \times \cdots \times C_{i-3}^{\varepsilon_{i}} \times C_{i-2} \times C_{i-2}^{-1} \times C_{i-2} \\
& \times C_{0} \times C_{0} \times C_{i+3}^{\varepsilon_{i+3}} \times C_{0} \times C_{i+5}^{\varepsilon_{i}+5} \times \cdots \times C_{n}^{\varepsilon_{n}}
\end{aligned}
$$

and clearlv

$$
\begin{aligned}
& C_{a} \cap A_{n-1}(X)=\left[C_{1}^{\varepsilon_{1}} \times \cdots \times C_{i-3}^{\varepsilon_{1-3}} \times \Delta_{i-2} \times C_{i-2}\right. \\
& \times C_{0} \times C_{0} \times C_{i+3}^{\xi+3} \times C_{0} \times C_{i+\xi}^{\xi_{1}+\xi} \times \cdots \times C_{n}^{\xi_{n}} \\
& \cup C_{1}^{\ell_{1}} \times \cdots \times C_{i-3}^{k_{1-3}} \times C_{i-2} \times \Delta_{i-2}^{-1} \\
& \times C_{0} \times C_{0} \times C_{i+3}^{\xi_{1}+3} \times C_{0} \times C_{i+\xi}^{\xi_{1}+\xi} \times \cdots \times C_{n}^{\xi_{n}} \\
& \cup C_{1}^{\varepsilon_{1}} \times \cdots \times C_{i-3}^{\varepsilon_{i-3}} \times C_{i-2} \times C_{i-2}^{-1} \times C_{i-2} \\
& \times e \times C_{0} \times C_{i+3}^{\varepsilon_{1}+3} \times C_{0} \times C_{i+\xi}^{\beta_{1}+\xi} \times \cdots \times C_{n}^{\varepsilon_{n}} \\
& \cup C_{1}^{\hat{1}_{1}} \times \cdots \times C_{i-3}^{\boldsymbol{k}_{-3}} \times C_{i-2} \times C_{i-2}^{-1} \times C_{i-2} \\
& \times C_{0} \times e \times C_{i+3}^{\beta_{1}+3} \times C_{0} \times C_{i+3}^{\beta_{1}+3} \times \cdots \times C_{n}^{\varepsilon_{n}} \\
& \cup C_{1}^{\varepsilon_{1}} \times \cdots \times C_{i-3}^{\varepsilon_{i-3}} \times C_{i-2} \times C_{i-2}^{-1} \times C_{i-2} \\
& \left.\times C_{0} \times C_{0} \times C_{i+3}^{\beta_{i}+3} \times e \times C_{i+3}^{\beta_{i}+\xi} \times \cdots \times C_{n}^{\varepsilon_{n}}\right] .
\end{aligned}
$$

Since $\Delta_{j}^{\xi}$ is homeomorphic to $C_{j}$, each of the terms in the above union is connected. Further as each of these terms also contains the point $a, C_{a} \cap$ $A_{n-1}(X)$ is connected. Therefore $C_{a} \cap A_{n-1}(X)$ is the component in $A_{n-1}(X)$ of $a$, as required.

In [10] Ordman showed that if $G$ and $H$ are arbitrary $k$-groups, then $\pi_{1}(G * H)=\pi_{1}(G) \times \pi_{1}(H) \times L$, where $G * H$ denotes the coproduct in the category of $k$-groups, and $L$ is some (unknown) group. He conjectured that $L$ 
is always trivial and showed that this is indeed true if $G$ and $H$ are countable CW-complexes with only one zero cell. In [9] and [12] it is shown that $G * H$ is homeomorphic to $G \times_{k} H \times_{k} \quad F(G \wedge H)$, where $G \wedge H$ is the smash product of $G$ and $H$; that is, $G \wedge H$ is the quotient space $G \times H / G \vee H$. So $\pi_{1}(G * H)=\pi_{1}(G) \times \pi_{1}(H) \times \pi_{1}(F(G \wedge H))$. Thus Ordman's problem is reduced to that of showing $\pi_{1}(F(G \wedge H))$ is always zero.

Corollary. If $X$ and $Y$ are path connected LEC $k$-Hausdorff $k$-spaces then $\pi_{1}(F(X \wedge Y))=0$. Hence, if $G$ and $H$ are path connected LEC $k$-groups, then $\pi_{1}(G * H)=\pi_{1}(G) \times \pi_{1}(H)$.

Proof We prove $X \wedge Y$ is simply connected and LEC, and then apply our theorem. Simple connectedness and LEC are respectively obtained from (1) and (2) by taking $Z=X \times Y$ and $A=X \vee Y$ :

Let $Z$ and $A$ be LEC $k$-Hausdorff $k$-spaces with $A$ a closed subspace of $Z$.

(1) If $Z$ and $A$ are path connected and the induced homomorphism $\pi_{1}\left(A, a_{0}\right) \rightarrow \pi_{1}\left(Z, a_{0}\right)$ is surjective then the quotient space $Z / A$ is simply connected.

(2) If the inclusion $A \subset Z$ is a closed cofibration then $Z / A$ is LEC.

(Observe that $\pi_{1}(X \vee Y)=\pi_{1}(X) * \pi_{1}(Y)$ and so $\pi_{1}\left(X \vee Y, a_{0}\right)$ $\rightarrow \pi_{1}\left(X \times Y, a_{0}\right)$ is surjective. Also $X \vee Y \rightarrow X \times Y$ is a closed cofibration, cf. [1, Theorem 2.7, p. 234].)

(1) is easily obtained from the Brown-van Kampen theorem and the proof of (2) is routine.

Acknowledgment. The authors are grateful to Professor R. Brown for helpful comments and, in particular, for pointing out (1) in the Corollary. The financial assistance of the United Kingdom Science Research Council is also gratefully acknowledged (S.R.C. Grant B/RG/3967).

\section{REFERENCES}

1. M. Boardman and R. M. Vogt, Homotopy invariant algebraic structures on topological spaces, Lecture Notes in Math., vol. 347, Springer-Verlag, Berlin and New York, 1973.

2. R. Brown, Groupoids and van Kampen's theorem, Proc. London Math. Soc. (3) 17 (1967), 385-401. MR 36 \#3345.

3. Elements of modern topology, McGraw-Hill, New York and London, 1968. MR 37 \#3563.

4. J. Dugundji, Topology, Allyn and Bacon, Boston, Mass., 1968. MR 33 \# 1824.

5. E. Dyer and S. Eilenberg, An adjunction theorem for locally equiconnected spaces, Pacific. J. Math. 41 (1975), 669-686.

6. M. I. Graev, Free topological groups, Izv. Akad. Nauk SSSR Ser. Mat. 12 (1948), 279-324; English transl., Amer. Math. Soc. Transl. (1) 8 (1962), 305-364. MR 10, 11.

7. J. P. L. Hardy, Topological groupoids: coverings and universal constructions, Ph. D. Thesis, University College of North Wales, Bangor, 1974.

8. P. J. Higgins, Notes on categories and groupoids, Van Nostrand Reinhold Math. Studies, no. 32, Van Nostrand Reinhold, London and New York, 1971. MR 48 \#6288.

9. S. A. Morris, E. T. Ordman and H. B. Thompson, The topology of free products of topological groups, Proc. Second International Group Theory Conference Canberra, 1973.

10. E. T. Ordman, Free products of topological groups which are $k_{\omega}$-spaces, Trans. Amer. Math. Soc. 191 (1974), 61-74.

11. appear). 
12. E. T. Ordman, Free products in the category of k-groups, General Topclogy and Appl.(to appear).

13. N. E. Steenrod, A convenient category of topological spaces, Michigan Math. J. 14 (1967), 133-152. MR $35 \# 970$.

School of Mathematics and Computer Science, University College of North Wales, BANGOR LI57 2UW, WALES

Current address (J. P. L. Hardy): 6 Gwyllt Road, Llanfairfechan, LL33 0EE, Gwynedd, United Kingdom

Current address (S. A. Morris): Department of Pure Mathematics, University of New South Wales, Kensington, N.S.W., Australia 